\title{
Leader Identification of Social Learning among Patients with Type 2 Diabetes Mellitus and Hypertension using Social Network Analysis
}

\author{
Rachata, N. ${ }^{1,3^{*}}$ and Chaikaew, N. ${ }^{2,3}$ \\ ${ }^{1}$ Business Computer, School of Information and Communication Technology, University of Phayao, Thailand \\ E-mail: napa.ra@up.ac.th \\ ${ }^{2}$ Geographic Information Science, School of Information and Communication Technology, University of \\ Phayao, Thailand, E-mail: nakarin.ch@up.ac.th \\ ${ }^{3}$ Research Unit of Spatial Innovation Development, School of Information and Communication Technology, \\ University of Phayao, Thailand \\ *Corresponding Author
}

DOI: https://doi.org/10.52939/ijg.v17i5.2011

\begin{abstract}
Preventing patients with type 2 diabetes mellitus and hypertension from cardiovascular complications is undeniably a public health problem that deserves research attention. The leader is important person for the social learning because the leader can provide valuable inspiration and knowledge to support a better quality of life for their community. Consequently, the patients can prevent themselves not to have complications with the presence of the leader. Furthermore, leader is a challenge for the social learning because the leader is changed under different conditions anytime. For these reasons, this paper proposes the analysis of a social learning for identifying leader among patients with type 2 diabetes mellitus and hypertension for preventing cardiovascular complications based on the social network analysis method. The proposed leader is expected that she/he is a role model who provides the ideal healthcare practice for other patients so they can learn healthcare practice from the leader for managing themselves appropriately throughout their healthcare journey. This proposed paper is proved with 189 patients with type 2 diabetes mellitus and hypertension in areas with high-risk patients. The experimental results show that the proposed leader has been widely accepted among the patients' social learning.
\end{abstract}

\section{Introduction}

Chronic diseases are major cause of the disabled and dreaded people globally. Additionally, they kill 41 million people each year (WHO, 2021) and account for 7 of every 10 deaths worldwide (WHO, 2014). Consequently, chronic diseases have become a leading cause that affects healthcare costs because these diseases generally have slow progression and take long duration for treatment. Cardiovascular diseases are chronic diseases and the number one health problem of death worldwide. They take an estimated 17.9 million people in 2016 and are expected to increase to 23.6 million in 2030 (WHO, 2017). Consequently, cardiovascular diseases are a serious public health problem worldwide because they affect large epidemiology, society, and economics. Generally, cardiovascular diseases deaths mostly take place in low-income and middleincome countries and with the people under the age of 70 years old. Diabetes mellitus is one of four main types of chronic diseases that kill 1.5 million people in 2019 (WHO, 2021).

Diabetes mellitus is a major cause of blindness, kidney failure, heart attacks, stroke, and lower limb amputation. Majority of patients with diabetes mellitus have type 2 diabetes mellitus. Most patients with type 2 diabetes mellitus are died from cardiovascular diseases (Sowers et al., 2001). The dead patients with type 2 diabetes mellitus have been attributed to hypertension due to it will make diabetes getting cardiovascular diseases faster (Sowers et al., 2001 and Campbell et al., 2011). Therefore, if the patients have type 2 diabetes mellitus and hypertension, the patients will have high risk for having cardiovascular complications. Moreover, cardiovascular complications are a significant health problem affecting the death of the patient with type 2 diabetes mellitus and hypertension worldwide. 
This evidence indicates that the prevention of patients with type 2 diabetes mellitus and hypertension from cardiovascular complications is a public health problem that deserves research attention. Social learning is learning process based on people observe attitudes, behavior, and consequences of those others' behaviors through their community interaction (Bandura, 2001 and Reed et al., 2010). People can learn new behavior with observation in their social environment and probably leads to behaviors or attitudes changing later. Hence, they can learn new positive behavior, that is the desired outcome, and they are likely to imitate and apply to their behaviors (Petrin, 2017). Nowadays, social learning in mobile environments, known as mobile learning, is presented that is outstanding learning, increasingly popular and affects many areas including healthcare, marketing, and education. For healthcare, majority of mobile learning is applied for enhancing the quality of personalized healthcare (Chorbev et al., 2011, Martínez-Pérez et al., 2013, Årsand et al., 2012, Triantafyllidis et al., 2013 and Rachata et al., 2018).

Meanwhile, the leader identification of a social learning is challenging. Instead of only share and learn activities in social learning, the leader identification activity should begin as well. Leader is one of the important skills that are the ability to influence, improve, and rally people in a community move toward the achievement of goals (Morgan, 2020). Furthermore, the leader can provide valuable inspiration and knowledge to support a better quality of life for their community. Consequently, the patients can prevent themselves not to have complications or worse health status or even to have a better health status in the presence of the leader. With the presence of the leader, it is a challenge for the social learning because the leader is changed under different conditions anytime. For the leader identification, many of the research works have proposed mainly frequency and volume of the participation (Sudweeks and Simoff, 2005 and Heckman and Misiolek, 2005), multi criteria decision making (Zendeh and Aali, 2011, Omar et al., 2015 and Muhisn et al., 2015), and network position of the leader (Temdee et al., 2006, Sutanto et al., 2011 and Knaub et al., 2018). One of the methods is visible and simple for identifying the leader, which is social network analysis method (Wasserman and Faust, 2012). Social network analysis is network position that presents patterns or regularities in structure relationship and provides information about relationship between the leader and members. Besides, social network analysis is appropriate to social and behavioral sciences.
This method is used various proposes (Temdee et al., 2006, Sutanto et al., 2011 and Knaub et al., 2018). Therefore, this paper proposes the analysis of a social learning for identifying leader among patients with type 2 diabetes mellitus and hypertension for preventing cardiovascular complications based on the social network analysis method. The proposed leader refers to a leader's behavior with expect that she/he is a role model who provides the ideal healthcare practice for other patients so they can learn healthcare practice from the leader for managing themselves appropriately throughout their healthcare journey. Moreover, the paper considers the frequency and the volume of participation with share and learns activities.

The paper is organized as follows. Section 2 proposes research methodology. Section 3 presents results and discussion. Finally, section 4 is the conclusion of this paper.

\section{Research Methodology}

The research methodology of this paper is shown in Figure 1 which includes data collection, model construction, and model validation.

\section{Data Collection}

Frequency and volume of sharing and learning engagement

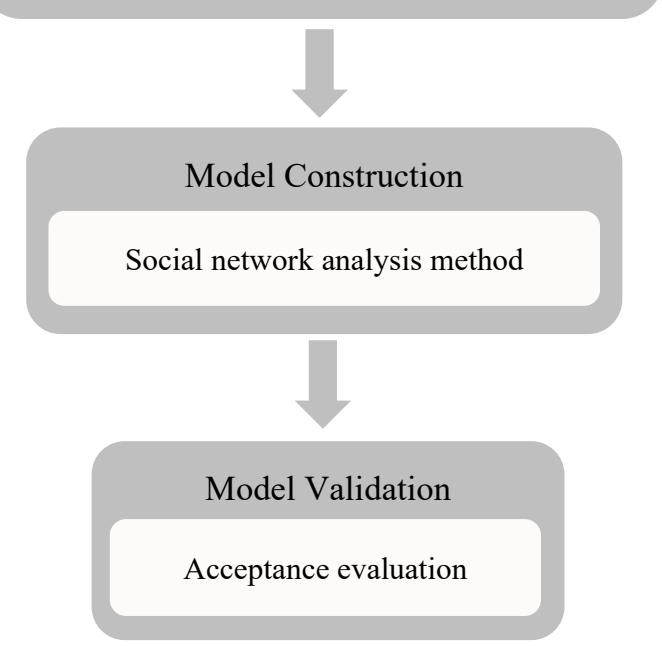

Figure 1: Research methodology of proposed leader identification

Firstly, data collection process consists of two events for constructing model. Two events are the frequency of sharing and learning engagement and the volume of sharing and learning engagement. 
Secondly, model construction process demonstrates leader identification among social learning of patients with type 2 diabetes mellitus and hypertension for preventing cardiovascular complications by using social network analysis method. Lastly, model validation process presents acceptance of leader identification result from these patients. The detail of the three processes is explained in a subsequent section.

\subsection{Data Collection}

The data collection in this paper is extension of proposed paper in Rachata et al., (2018). Data were divided into the frequency of sharing and learning engagement and the volume of sharing and learning engagement. Frequency of engagement means how often the numbers of time that the patients shared their own experiences and learned from other experiences. Meanwhile, volume of engagement means amount of the numbers of time that the patients shared their own experiences and learned from other experiences in each day. Besides, data were collected with 189 patients with type 2 diabetes mellitus and hypertension in areas with high-risk patients of Chiang Rai province, which is Mae Chan district and Mae Lao district, for six months by using the mobile application. Details of patients are 102 males and 87 females aging between 35-67 years old and having type 2 diabetes mellitus and hypertension. They are mostly contractors, merchants, and farmers and having medium technical skills. The protocol, which was used to select the patients to join this research, has been approved by Mae Fah Luang University Research Ethics Committee (No. 041/2560).

Figure 2 shows cartography of the number of 189 patients with type 2 diabetes mellitus and hypertension case studies in one sub-district of Mae Chan district, which includes Pa Tueng, and five sub-districts of Mae Lao district, which includes Pong Phrae, Pa Ko Dam, Bua Sali, Dong Ma Da, and Chom Mok Kaeo. There are five classes which are used for representing different classes with the presence of the numbers of patient cases by using Jenks natural breaks classification method (Jenks, 1967). The method is a data clustering method by determining the best arrangement of values into different classes. The process of this method is to seek to reduce the variance within classes and maximize the variance between classes. Five classes consist of the number of patients with type 2 diabetes mellitus and hypertension cases between 1 and 16 means "very low" cases, between 17 and 29 means "low" cases, between 30 and 31 means "moderate" cases, between 32 and 36 means "high" cases, and between 37 and 47 means "very high" cases, respectively.
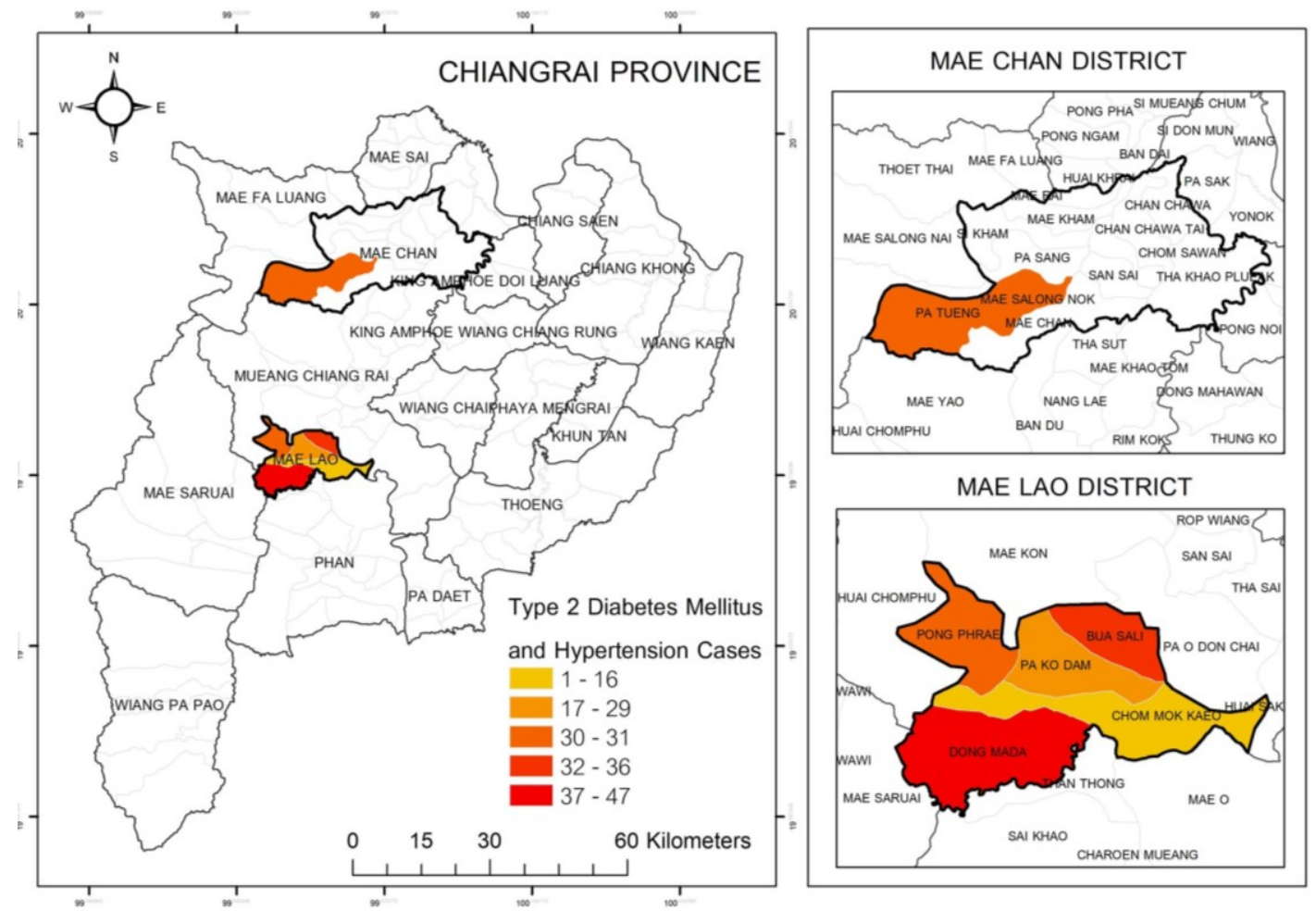

Figure 2: Cartography of the number of 189 patients with type 2 diabetes mellitus and hypertension case studies in sub-district of Mae Chan district and Mae Lao district 


\subsection{Model Construction}

The model construction used social network analysis method and data collection for analyzing of a social learning for the purpose of leader identification among social learning of patients with type 2 diabetes mellitus and hypertension for preventing cardiovascular complications. There are two sections of social network analysis method are used for constructing model, which are network positions and measurements.

\subsubsection{Network positions of social network analysis method}

Network positions of social network analysis method consist of three network positions for identifying the leader. Furthermore, the network positions focus on a non-direction network.

\section{- Position Centrally in the Network (Highest Power and Response):}

The leader should be the position center in the network. This position presents the leader has the highest interest and behaviour may be desired.

\section{- Position Averagely Close to Many Other Patients (Quickly Interaction):}

The leader should be position averagely close to many other patients to reach other patients quickly.

\section{- Position Lies between Many Unconnected (Influence):}

The leader should be position lies between many unconnected patients. Many of the communication between unconnected patients present their influence. Accordingly, there is a high chance to connect those patients.

\subsubsection{Measurements of social network analysis method}

Measurements of social network analysis method consist of three measurements for describing mentioned three network positions. In addition, the proposed social learning among patients with type 2 diabetes mellitus and hypertension is surveyed as a social network based on social network analysis method, which is patients are connected by a set of co-sharing/learning relationships.

\section{- Degree of Centrality:}

Degree of centrality presents the leader's level of popularity in the community. The leader should has a high degree of centrality. In this paper, the degree of centrality as the following equation (Wasserman and Faust, 2012):

$$
D(i)=\frac{d(i)}{\sum d(i)}
$$

Equation 1

where: $d(i)=$ degree of a patient

$$
\begin{aligned}
\sum d(i)= & \text { total degree of a patient } \\
& \text { (total connections) }
\end{aligned}
$$

\section{- Closeness:}

Closeness presents the patient's closeness to the other patients in the community. Leaders are central if they can interact quickly with others, so they should be team patients with high closeness. This ensures that information reaches the majority of team patients quickly. In this paper, the closeness as the following equation (Wasserman and Faust, 2012):

$$
C(i)=\frac{n-1}{\sum_{j \in U} d(i, j)}
$$

Equation 2

where: $d(i, j)=$ shortest path between a patient $\mathrm{i}$ and a patient $\mathrm{j}$

$$
\begin{aligned}
& U=\text { set of all patients } \\
& n=\text { total number of patients }
\end{aligned}
$$

\section{- Betweenness:}

Betweenness presents the patient who has been between many unconnected patients. So, the leader should have a betweenness with a high value. In this paper, the closeness as the following equation (Wasserman and Faust, 2012):

$$
B(i)=\frac{2}{(n-1)(n-2)} \sum_{j<k} \frac{g_{j k}(i)}{g_{j k}}
$$

Equation 3

where:

$g_{j k}(i)=$ number of shortest paths between $\mathrm{j}$ and $\mathrm{k}$ through i

$g_{j k}=$ number of the shortest paths between $\mathrm{j}$ and $\mathrm{k}$

$n=$ total number of nodes (patients)

Thus, the proposed leader identification as following equation:

$$
L_{i}=\sqrt{D(i)^{2}+C(i)^{2}+B(i)^{2}}
$$

Equation 4

where: $D(i)=$ degree of centrality

$$
\begin{aligned}
& C(i)=\text { closeness } \\
& \mathrm{B}(\mathrm{i})=\text { betweenness }
\end{aligned}
$$

Finally, the leader is the maximum value of leader identification. 


\subsection{Model Validation}

The model validation consists of two validation results which include leader identification and evaluation of leader identification. The results of identifying leader with three measurements of social network analysis method. Meanwhile, the results of evaluation of leader identification with measuring how much patients agree on results of identifying leader for their community. The proposed paper is proved with 189 patients with type 2 diabetes mellitus and hypertension in Mae Chan district and Mae Lao district, Chiang Rai province for six months.

\section{Results and Discussion}

\subsection{Leader Identification}

The results from the experiment are shown in Table 1 , that is example of social learning based on social network analysis method for identifying leader among 189 patients with type 2 diabetes mellitus and hypertension. For example, Table 1 shows social learning data at the end of six months.

Table 1: Example of social network analysis method for identifying leader

\begin{tabular}{|c|c|c|c|c|}
\hline \multirow{2}{*}{$\begin{array}{c}\text { Patient } \\
\text { ID. }\end{array}$} & \multicolumn{4}{|c|}{$\begin{array}{c}\text { Measurements at the end of } \\
\text { six months }\end{array}$} \\
\cline { 2 - 5 } & D(i) & C(i) & B(i) & L(i) \\
\hline 01 & 0.76 & 0.81 & 2.44 & 2.68 \\
\hline 02 & 0.34 & 0.00 & 0.00 & 0.34 \\
\hline 03 & 0.39 & 0.00 & 0.98 & 1.05 \\
\hline 04 & 0.00 & 0.00 & 0.49 & 0.49 \\
\hline 05 & 0.81 & 0.00 & 0.81 & 1.15 \\
\hline 06 & 0.00 & 0.00 & 0.49 & 0.49 \\
\hline 07 & 0.54 & 2.44 & 0.29 & 2.52 \\
\hline 08 & 1.22 & 1.22 & 1.22 & 2.11 \\
\hline 09 & 0.00 & 1.64 & 0.00 & 1.64 \\
\hline 10 & 0.34 & 0.81 & 0.00 & 0.88 \\
\hline 11 & 0.34 & 0.00 & 0.00 & 0.34 \\
\hline 12 & 0.34 & 0.00 & 0.15 & 0.37 \\
\hline 13 & 0.42 & 2.44 & 0.00 & 2.48 \\
\hline 14 & 0.88 & 0.81 & 2.74 & 2.99 \\
\hline 15 & 0.34 & 0.98 & 0.15 & 1.05 \\
\hline 16 & 0.51 & 0.20 & 1.96 & 2.04 \\
\hline 17 & 0.86 & 1.42 & 2.44 & 2.95 \\
\hline 18 & 0.44 & 0.20 & 1.96 & 2.02 \\
\hline 19 & 0.76 & 0.00 & 0.81 & 1.11 \\
\hline 20 & 0.42 & 0.81 & 0.24 & 0.94 \\
\hline & & & & \\
\hline
\end{tabular}

Leader identification presents three measurements of social network analysis method. These results consist of other patients learn from a patient (D(i)), a patient shares to other patients (C(i)), known and unknown patient in the community (B(i)). Finally, leader appeared in social learning (L(i)) with maximum value. The experimental results present that patient ID 14 is the leader because s/he has the maximum value of leader identification $(\mathrm{L}=2.99)$. Accordingly, the proposed patient ID 14 is expected that she/he is a role model who provides the ideal healthcare practice for other patients.

\subsection{Evaluation of Leader Identification}

The evaluation results are to confirm obtained leader of social learning with measuring how much 189 patients agree on results of identifying leader for their community. That is number of leadership votes received to be a role model who provides the ideal healthcare practice for them and interesting to learn knowledge and motivation from a leader. The results from the evaluation are shown in Table 2.

Table 2: Example of evaluation of leader identification

\begin{tabular}{|c|c|}
\hline $\begin{array}{c}\text { Patient } \\
\text { ID. }\end{array}$ & $\begin{array}{c}\text { Number of leadership votes } \\
\text { received (V(i)) }\end{array}$ \\
\hline 01 & 2 \\
\hline 02 & 0 \\
\hline 03 & 1 \\
\hline 04 & 0 \\
\hline 05 & 1 \\
\hline 06 & 0 \\
\hline 07 & 2 \\
\hline 08 & 1 \\
\hline 09 & 1 \\
\hline 10 & 1 \\
\hline 11 & 0 \\
\hline 12 & 0 \\
\hline 13 & 1 \\
\hline 14 & 3 \\
\hline 15 & 1 \\
\hline 16 & 1 \\
\hline 17 & 2 \\
\hline 18 & 1 \\
\hline 19 & 1 \\
\hline 20 & 1 \\
\hline & \\
\hline
\end{tabular}

For example, Table 2 shows the number of votes received (V(i)) from social learning. It can be confirmed that the proposed leader, who is patient ID 14, has been widely accepted among the patients' social learning because $\mathrm{s} / \mathrm{he}$ received most voted from their community $\left(\mathrm{V}_{\mathrm{i}}=3\right.$ patients from 20 patients).

From both results, the results show that the leader identification is measured reasonably from the 
social network analysis method and the patient who becomes a leader is accepted reasonably among the patients' social learning. Furthermore, future work will focus on more patient cases and periods to achieve more intensive leader identification. Moreover, monitoring of social learning with the presence of the leader and role model is the consideration for future work. More importantly, the proposed leader identification is also expected to study among patients with other chronic diseases.

\section{Conclusion}

This paper proposes a social network analysis-based method for identifying leader of social learning among patients with type 2 diabetes mellitus and hypertension for preventing cardiovascular complications. The proposed identification uses three measurements of social network analysis method which includes degree of centrality, closeness, and betweenness. This identification is constructed with the frequency of sharing and learning engagement and the volume of sharing and learning engagement. The experimental results show that the proposed leader has been widely accepted among the patients' social learning. The future work will be focusing on increasing number of the patient cases and number of periods to achieve more intensive leader identification. Moreover, monitoring of social learning with the presence of the leader and role model is consideration.

\section{References}

Årsand, E., Frøisland, D. H., Skrøvseth, S. O., Chomutare, T., Tatara, N., Hartvigsen, G. and Tufano, J. T., 2012, Mobile Health Applications to Assist Patients with Diabetes: Lessons Learned and Design Implications. J. Diabetes Sci. Technol., Vol. 6(5), 1197-1206.

Bandura, A., 2001, Social Cognitive theory: An Agentic Perspective. Annu. Rev. Psychol, Vol. 52(1), 1-26.

Campbell, N. R. C., Gilbert, R. E., Leiter, L. A., Larochelle, P., Tobe, S., Chockalingam, A., Ward, R., Morris, D., Tsuyuki, R. T. and Harris, S. B., 2011, Hypertension in People with Type 2 Diabetes Update on Pharmacologic Management. CFP, Vol. 57(9), 997-1002.

Chorbev, I., Sotirovska, M. and Mihajlov, D., 2011, Virtual Communities for Diabetes Chronic Disease Healthcare. Int. J. Telemed. Appl., 1-7. DOI:10.1155/2011/721654.
Heckman, R. and Misiolek, N. I., 2005, Leaders and Followers in Student Online Project Teams. Proceedings of the 38th Hawaii International Conference on System Sciences, IEEE. http://www.who.int/mediacentre/factsheets/fs35 $5 / \mathrm{en} /$

Jenks, G. F., 1967, The Data Model Concept in Statistical Mapping, International Yearbook of Cartography, Vol. 7, 186-190.

Knaub, A. V., Henderson, C. and Fisher, K. Q., 2018, Finding the Leaders: an Examination of Social Network Analysis and Leadership Identification in STEM Education Change. International Journal of STEM Education, Vol. 5(1), 1-14.

Martínez-Pérez, B., De La Torre-Díez, I., LópezCoronado, M. and Herreros-González, J., 2013, Mobile apps in Cardiology. JMIR MHealth UHealth, Vol. 1(2). 1-15.

Morgan, J., 2020, What is Leadership, and who is a leader?. https://www.chieflearningofficer.com/2020/01/06/what-is-leadership-and-who-is-aleader/.

Muhisn, Z. A. A., Omar, M., Ahmad, M. and Muhisn, S. A., 2015, Team Leader Selection by Using an Analytic Hierarchy Process (AHP) Technique. JSW, Vol. 10(10), 1216-1227.

Omar, M., Aljasim, Z. A., Ahmad, M., Baharom, F., Yasin, A., Mohd, H. and Muhd Darus, N., 2015, Team formation model of selecting team leader: An analytic hierarchy process (AHP) approach. ARPN Journal of Engineering and Applied Sciences. Vol. 10(3), 1061-1067.

Petrin, R., 2017, What is Social Learning? http://www.managementmentors.com/about/corp orate-mentoring-matters-blog/bid/52304/whatis-social-learning.

Rachata, N., Szabó, Z. and Temdee, P., 2018, Myhealth: Mobile Application Supporting social learning among Patients with Type 2 Diabetes Mellitus and Hypertension. International Journal of Applied, Vol. 11(2). 6-15.

Reed, M. S., Evely, A. C., Cundill, G., Fazey, L., Glass, J., Laing, A., Newig, J., Parrish, B., Prell, C., Raymond, C. and Stringer, L. C., 2010, What is Social Learning?. Ecol. Soc., Vol. 15(4). 1-10, DOI: 10.5751/ES-03564-1504r01.

Sowers, J. R., Epstein, M. and Frohlich, E. D., 2001, Diabetes, Hypertension, and Cardiovascular Disease: an Update. AHA Journals: Hypertension, Vol. 37(4), 1053-1059. 
Sudweeks, F. and Simoff, S. J., January 2005, Leading Conversations: Communication Behaviours Of Emergent Leaders In Virtual Teams. In proceedings of the 38th annual Hawaii international conference on system sciences, IEEE. DOI:10.1109/HICSS.2005.383

Sutanto, J., Tan, C. H., Battistini, B. and Phang, C. W., 2011, Emergent Leadership in Virtual Collaboration Settings: A Social Network Analysis Approach. Long Range Planning, Vol. 44(5-6), 421-439.

Temdee, P., Thipakorn, B., Sirinaovakul, B. and Schelhowe, H., April 2006, Of Collaborative Learning Team: An Approach for Emergent Leadership Roles Identification by Using Social Network Analysis. In International Conference on Technologies for E-Learning and Digital Entertainment, Springer, Berlin, Heidelberg, 745-754.

Triantafyllidis, A. K., Koutkias, V. G., Chouvarda, I. and Maglaveras, N., 2013, A Pervasive Health System Integrating Patient Monitoring, Status Logging, and Social Sharing. IEEE J. Biomed. Health Inform, 17(1), 30-37.
Wasserman, S. and Faust, K., 2012, Social Network Analysis Method and Applications, Cambridge: University of Cambridge).

World Health Organization, 2014, Noncommunicable Diseases Country Profiles 2014, (WHO: Geneva).

World Health Organization, 2017, Cardiovascular Diseases. https://www.who.int/news-room/factsheets/detail/cardiovascular-diseases-(cvds).

World Health Organization, 2021, Diabetes. https://www.who.int/news-room/factsheets/detail/diabetes

World Health Organization, 2021, Noncommunicable diseases". https://www.who.int/news-room/factsheets/detail/noncommunicable-diseases

Zendeh, A. B. and Aali, S., 2011, An AHP Approach for Selecting the Suitable Leadership Style. Int. Proc. Econ. Dev. Res, Vol. 25, 20-24. 